\title{
Radiation Therapy for Breast Cancer and Clonal Chromosome Translocations: A Fluorescence In Situ Hybridization Study
}

\author{
Marcelo L. Larramendy, Päivi Majander, Tiina Saarto, Mikko Tenhunen, \\ Rosa Caballín, Inkeri Elomaa, and Sakari Knuutila
}

\begin{abstract}
The frequency of chromosomal translocations was analyzed in skin fibroblast cell cultures derived from irradiated and nonirradiated skin biopsies from five cases of breast adenocarcinoma in women, who had undergone radiotherapy after surgery. The study was performed at the first annual check-up. Chromosomal in situ suppression (CISS) hybridization was performed using metaphase nonisotopic fluorescence in situ hybridization (FISH) with library probes specific for chromosomes 1, 2 , $3,4,5,7,8,13,19$, and 20. The results showed that the frequency of clonal translocations in metaphases obtained from irradiated areas was significantly higher than in metaphases from the nonirradiated tissue samples. (Olsevier Science Inc., 1998
\end{abstract}

\section{INTRODUCTION}

Postoperative radiation is a commonly used treatment for patients with breast cancer [1]. Radiation therapy exerts both acute and chronic effects on normal tissue included within treatment fields $|2|$. Although irradiation confined to the breast has only rarely been associated with secondary malignancies, the risk of soft tissue sarcoma has been reported with an actual incidence of $0.2 \%$ at 10 years $[2-4]$. Contralateral breast cancers (from scatter irradiation because of treatment of the opposite breast) have been observed to occur at rates from $0.5 \%$ to $0.9 \%$ per year after orthovoltage treatment [2].

In breast cancer patients the effects of concomitant adjuvant chemotherapy and irradiation on the induction of secondary malignancies are not known yet. The incidence. may increase within a longer observation period, as documented in a recent report of a cohort of 1,380 patients treated for childhood Hodgkin disease [5]. In this study the estimated cumulative risk of a second cancer was $7 \%$,

From the Departments of Medical Genetics (M. L. L., S. K.) and Virology (P. M.). Haartman Institute, University of Helsinki. Helsinki. Finland; Laboratorio de Citogenética v Cátedra de Citologia, Facultad de Ciencias Naturales, Universidad Nacional de La Plata (M. L. L.). La Plata, Argentina; Department of Oncology. Helsinki University Central Hospital (T. S., M. T., I. E.). Helsinki. Finland; and Departament de Biologia Animal y Vegetal, Universitat Autónoma de Barcelona (R. C.), Barcelona. Spain.

Address reprint requests to: Sakari Knuutila, Ph.D. Department of Medical Genetics, Haartman Institute, P.O. Box 21 (Hoartmaninkatu 3), FIN-00014 Helsinki. Finland.

Received October 30, 1996; accepted February 17, 1997.
15 years after the initial diagnosis. Breast cancer was the most common solid tumor in this group of patients. The majority of breast cancers arose within the field of radiation with doses higher than $20 \mathrm{~Gy}$ in the mantle region [5].

Substantial evidence shows that ionizing radiation leads to cell damage caused by DNA double strand breaks, which may later be manifested as chromosomal aberrations $[6,7]$. Coll death, accordingly, appears to result from the loss of genetic material through production of acentric fragments accompanied by unstable aberrations, such as dicentric and ring chromosomes [8-12]. Chromosomal aberrations are widely used when monitoring genetic alterations induced by irradiation [13]. The presence of dicentric chromosomes on phytohemagglutinin (PHA) stimulated lymphocyte cultures is applied to grading of chromosomal aberrations induced by irradiation. A drawback in this approach is that PHA affects only lymphocytes. Another linitation is the instability of these aberrations: cells that carry a dicentric chromosome disappear in subsequent mitoses $[9,14]$, though it is assumed that radiation-induced reciprocal translocations and dicentrics in lymphocytes are formed in an equal proportion of $1: 1$ [15]. Moreover, the assay does not readily lend itself to the study of clonal chromosomal aberrations, such as a translocation frequently found in malignant tumors [16]. A translocation assay has not been routinely used in previous studies by chromosomal banding, because it is laborious when applied to the scoring of this type of aberration. Recent chromosomal painting techniques with chromosome-specific probes have been used efficiently for large-scale screening of translocations. 
To evaluate the frequency of chromosomal aberrations in irradiated skin, we used a chromosomal painting technique to score the translocations in skin samples from breast cancer patients treated with irradiation and compared the scores obtained from irradiated areas and nonirradiated areas.

\section{MATERIALS AND METHODS}

\section{Patients, Therapy Regimen, and Sampling Schedule}

The donors were five women with operable breast carcinoma and histologically proved axillary metastases. treated between November 1992 and February 1993 at the Department of Oncology. Helsinki Iniversity Central Hospital. All patients were operated with axillary ovacuation. Characteristics of the patients and their treatment plans are given in lable 1. Following mastectomy the chest wall was irradiated from oblique field using electron beams. After breast conserving resection the whole broast was irradiated with lateral and medial tangential portals using 6 MV photon energy. A boost to the tumor site was given using electron energy to a total dose of 10 Gy in five consecutive fractions. Supraclavicular and axillary fields were irradiated using $6 \mathrm{MV}$ photon energy and parasternal area using $12 \mathrm{MeV}$ electron energy. At the end of the radiotherapy, the mean dose to the skin was 41 Gy (37-42 (iv) ('able 1). Radiotherapy was given simultaneously with adjuvant therapy. At the first annual check-up, skin biopsies were taken from the irradiated field $3 \mathrm{~cm}$ above the operative scar at the mean clavicular line. The control biopsies were taken from the contralateral breast at the same horizontal line and vertically at the mean clavicular line.

\section{Breast Fibroblast Cell Cultures}

Cultured fibroblasts grown from irradiated skin biopsies and nonirradiated control skin biopsies from breast-cancer patients were analyzed. The fibroblast cultures were set up according to conventional skin culture procedures in supplemented culture medium $180 \%$ RPMI 1640 (GIBCO (Frand Island, NY, USA), 10\%-15\% fetal bovine serum (GIBCO), $0.29 \mathrm{mg} / \mathrm{ml}$ L-glutamine (GIBCO). 100 units/ml penicillin (CIBCO) and $100 \mathrm{mg} / \mathrm{ml}$ streptomycin (CIBCO)). In all samples, the molecular cytogenetic analysis of metaphase fibroblasts was performed on exponentially growing cells in passages 3 to 10 after an overnight Colcenid treatment (0.1 mg/ml; (jIBCO), conventional harvest with KC:I incubation (0.075 M. $\left.37^{\circ} \mathrm{C} .10 \mathrm{~min}\right)$, and methanolacetic acid (3:1) fixation. Slides were processed according 10 in situ hybridization procedures (see below) after air-drying for at least 18 hours.

\section{Chromosomal Painting By Chromosomal In Situ Suppression (CISS) Hybridization Using Chromosome-Specific Library Probes}

Hybridizations were carried out with DNA from bacteriophage library probes established from sorted human chromosome 1 (L.ibrary LA, American Type Culture collection, ATC, Rockville. MD, LSA), chromosome 2 (ATCC; LL02NSO1), chromosome 3 (ATCC, LA03NSO2. chromosome? 4 (ATCC: LLO4NSO2), chromosome 5 (ATC: IAO5NSO1), chromosome 7 (A'CC) LAO7NSO1), chromosome 8 (ATC LI.08NS02), chromosome 13 (ATCC. LL13NSO2), chromosome 19 (AlCC: LI.19NSO1), and chromosome 20 (ATCC: IL20NSO1) from pter to qter. The probes were labeled by nick-translation using biotin-11-dtJT' (Sigma Chemical (o), St. I.ouis. MO. IISA) according to the instructions of the kit supplier (Vick-translation Kit. Bethesda Research Laboratories, Bethesda, MI), (ISA). (ISS hybridization and detection of the hybridized probes with fluorescein isothiocVanate (FITC) conjugated avidin (Vector Laboratories, Burlingame, (A, USA) was performed as described in detail elsewhere [17|. The signal was amplified as described by Pinkel et al. |18|. The cells were counterstained with $4^{\prime}$,6diamidino-2-phenylindole-dihydrochloride (DAPI. Sigma) and propidium iodide (Sigma). The signals were prevented from fading with Vectashield mounting medium (Vector) and analyzed using a Zeiss Laborlux fluorescence

Table 1 Patients and treatment characteristics

\begin{tabular}{|c|c|c|c|c|c|}
\hline Donors & 1 & 2 & 3 & 4 & 5 \\
\hline Menopausal status & Premenop. & Postmenop. & Postmenop. & Promenop. & Premenops. \\
\hline Age at biopsy $(\mathrm{yr})$ & 53 & 6.3 & 67 & 47 & 45 \\
\hline INM & T2.N1M0 & T2N1M0 & T2N1Mo & T2N1MO & $\mathrm{T} 2 \mathrm{~N} 1 \mathrm{MO}$ \\
\hline Histology & Inobular & Ductal & Ductal & Durctal & I)uctal \\
\hline (Operation & Mastectomy & Mastectomy & Resection & Mastectonly & Mastectomy \\
\hline Systemic: therapy & $(: \mathrm{Ml}$ & Toremifene: & Toremitene: & CMF + 'lamoxifen & C:Mr' \\
\hline RT total/daily dose (Cy) & $50 / 2$ & $50 / 2$ & $50+101 / 2^{i}$ & $52 / 2$ & $50 / 2$ \\
\hline R'T energy & E6 MeV & $\because 6 \mathrm{MeV}$ & $\mathrm{FG}_{\mathrm{G}} \mathrm{MV}$ & IG MeV & EG MeV \\
\hline R'I skin dose (civ) & 40 & 42 & 37 & 42 & 42 \\
\hline Skin reaction after RT & Erythema & Fruthena & Lirvthema & Eirythema & Lirvthema \\
\hline Time: from RT to biopsy & $43 \mathrm{wk}$ & $39 \mathrm{wk}$ & $40 w k$ & $31 \mathrm{wk}$ & $41 w k$ \\
\hline Site of metastases & $\mathrm{N}(1$ & $N_{0}$ & No & $N_{()}$ & Skin, liver ${ }^{i}$ \\
\hline Current status at $4 y r$ & Alive: & Alive? & Alive & Alive? & Death \\
\hline
\end{tabular}



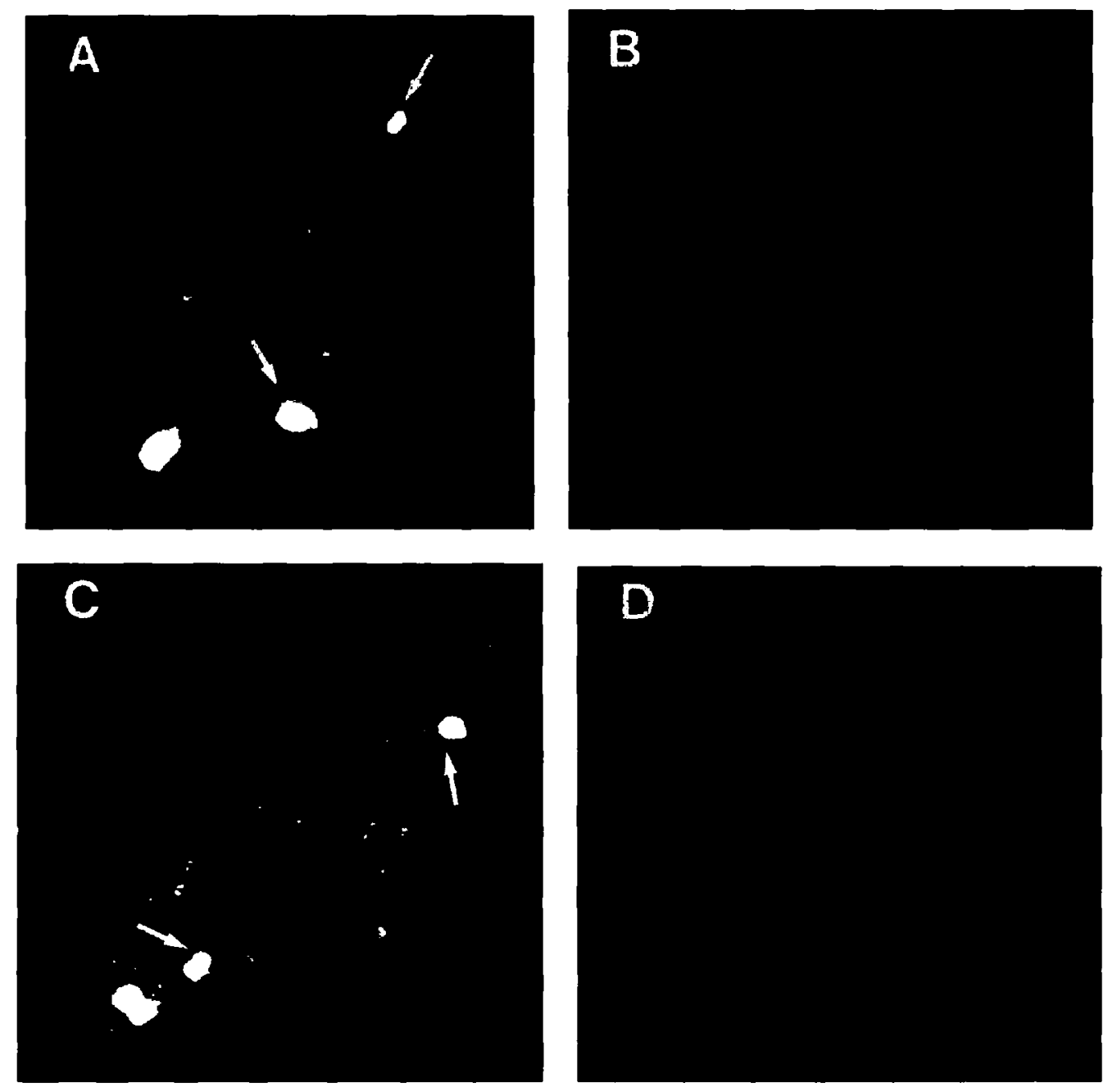

Figure 1 Metaphase chromosomes in fibroblasts from irradiated skin biopsies from patient $1(A-B)$ and patient 3 (C-I) after CISS hybridization with biotinylated chromosome 13- (LL13NSO2) or 20- (LL20NSO1) specific library IDNA probes, respectively, detected with avidin-FITC (fluorescein isothiocyanate) and propidium iodide chromosome counterstaining $(\Lambda, C) ; B$ and $D$. same metaphase spreads as in $\Lambda$ and $C$, respectively, after 4;6-diamidino2phenylindole (I)API) chromosome counterstaining. The arrows indicate how one look at the painting assay gives a reliable demonstration of the translocation.

photomicroscope equipped with Zeiss filters 02 and 09 for FITC, and DAPI fluorochromes, respectively.

\section{Cytogenetic Analysis}

The slides were coded and analyzed in a donble-blind manner by one observer. The number of hybridization signals and the presence of structural translocations was evaluated and determined by analyzing at least 200 metaphase spreads per sample, and expressed as the number of translocations per 100 cells. When identical chromosomal translocations were observed in more than $2.0 \%$ of the metaphases, the translocation was considered to be of clonal origin. In these cases only a tentative determination by the morphologic features of the chromosomes or chromosomal groups involved was achieved. When necessary, photographs were taken on Kodak Ektachrome 400 ASA color slide film.

\section{Statistical Analysis}

The statistical significance of the results was tested by means of the paired t-test and '2, using the StatWorks software (Heyden and Son, London, UK). P-values were given for the differences in mean frequencies. Differences yielding a p-value $<0.05$ were considered statistically significant.

\section{RESULTS}

The frequency of nonclonal chromosome translocations was equal both in irradiated and control specimens, whereas the frequency of clonal translocations was significantly higher in irradiated specimens $(\mathrm{p}<0.001)$ (Table 2$)$. Moreover, the frequency of nonclonal translocations affecting chromosomes $2,3,4$, and 13 was much higher in the irradiated samples than in the controls $(p<0.05)$. No clonal translocations were found in the nonirradiated specimens. 





In all cases, except donor 4 , the irradiated samples contained clonal chromosomal translocations affecting chromosomes 1 (donors 1 and 5), 2 (donors 1 and 2), 3 (donor 5), 4 (donors 2 and 5), 7 (donor 5), 13 (donor 3), 19 (donor 1), and 20 (donor 1$)(p>0.05)$.

\section{DISCUSSION}

After in situ hybridization with chromosome-specific library probes, complete individual chromosomes and the translocation involved could easily be identified (Fig. 1). The size, centromere index, and DAPI staining pattern of a chromosome designated as "complete" (no translocations) was apparently normal. Despite this designation and a seemingly normal state, these complete chromosomes may contain fine structural aberrations, the existence of which can only be ruled out by additional investigations. Because chromosomal painting does not verify the precise breakpoints of the translocated chromosomes, it may be still possible that some of the clonal translocations we found represent nonclonal changes. To minimize the number of false positive results we decided to change the ISCN [19] definition criteria for a clone from two similar cells per any number of cells studied to four similar cells in 200 colls analyzed. Comparison between metaphase painting and G-banding analysis shows that the former is essentially faster and interpretations of complicated translocations are easier. Besides, the metaphases to be scored need not be solected.

Chromosome painting also reveals chromosomal aberrations other than translocations, for example ring chromosomes, and dicentric and acentric fragments. As these aberrations were only seen in a single metaphase cell, the aberrations presented in detail in the present report are the translocations.

We found clonal translocations in the irradiated specimens but not in the nonirradiated specimens. Even though some of the changes may be clonal aberrations caused by culture conditions in vitro, the dramatic difference in the frequencies of these aberrations may indicate that the irradiation had induced clonal translocations in vivo. Our observations agree well with the findings made by chromosome banding analysis [20] showing the presence of cells with sequential changes. As Savage and Bigger [20] observed the clonal changes in various parts of the biopsy, the aberrations could not be culture artifacts. Thus our results as well as those previously reported [20] clearly indicate in vivo cell division of the nucleated cells after the therapy regimen.

The nonirradiated specimens showed a seemingly high number of nonclonal aberrations. Because no nonirradiated healthy controls were selected, these results cannot be reliably verified. In irradiated areas the frequency of nonclonal translocations involving chromosomes $2,3,4$, and 13 was higher than in nonirradiated controls. As the highest number of cells studied was 200 , it is still possible that some of these translocations may be clonal.

In human cancer, some 100 recurrent translocations have been described [16] and more than 50 of them are known to activate cellular oncogenes [21]. Translocations are thus an essential mechanism in the activation of can- cer genes. Before our finding can be used in the evaluation of the risk of secondary malignancies in certain patients, studies of a larger series of patients with longer follow-up periods are required. At present, however, our results highlight the technical value of translocation painting in efforts to reveal the genetic alterations liable to induce cancer.

\section{REFERENCES}

1. Mansfield CM, Krishnan I, Komarnicky LT, Ayvangar KM Kramer CA (1991): A review of the role of radiation therapy in the treatment of patients with breast cancer. Sem Oncol 18:525-535.

2. Kurtz JM, Mirabell R (1992): Radiation therapy and breast conservation: Cosmetic results and complications. Semin Radiat Oncol 2:125-131.

3. Bernstein EP, Sullivan FJ. Mitchell JB, Salomon (iD, Glatstein E (1993): Biology of chronic: radiation effect on tissues and wound healing. Clin Plast Surg 20:435-453.

4. Robinson E, Neugut AL, Wylie P (1988): Cilinical aspects of postirradiation sarcomas. J Natl Cancer Inst 80:233-240.

5. Bhatia S, Rolninson LL, Oberlin O. Creenbern M, Bunin (; Fossati-Bellani F, Meadows AT (1996): Breast cancer and other second neoplasms after childhood Hodgkin's disease. $N$ Engl J Med 3:34:745-751.

6. Natarajan $\triangle \mathrm{T}$. Obe (; (1978): Molecular mechanisms involved in the production of chromosomal aberrations. Mutation Res 52:137-149.

7. Wlodek D, Hittelman WN (1988): 'The relationship of DNA and chromosome damage to survival of synchronized $x$-irradiated L5178Y colls. Radiat Ress 115:550-565.

8. Bedford JS, Mitchell JB, Griggs $\mathrm{HC}^{\circ}$, Bender MA (1978): Radiation-induced cellular reproductive death and chromosome aberrations. Radiat Res 76:573-586.

9. Bianchi NO, Bianchi MS, Larramendy ML (1979): Kinetics of human lymphocyte division and chromosomal radiosensitivity. Mutation Res 63:317-324.

10. Carrano $\Lambda V$ (1973): Chromosome aberrations and radiationinduced cell death. II. Predicted and observed cell survival. Mutation Res 17:355-359.

11. Carrano AV (1973): Chromosome aberrations and radiationinduced cell death. I. Iransmission and survival parameters of aberrations. Mutation Res 17:341-353.

12. Pohl-Rüling J, fischer P. Hais (), Obe G, Natarajan AT, van Buul PP, Buckton KE, Bianchi NO, I arramendy ML, Kucerova M, Polikova Z, leonard A, Fabry I., Paliti F. Sharma T. Bender W. Mukherjee RN. Mukherjee U (1983): Effect of low dose acute $\mathrm{X}$-irradiation on the frequencies of chromosomal aberrations in human peripheral lymphocytes in vitro. Mutation Res 1:71-82.

13. Revell SH (1983): Relationship between chromosomal damage and cell death. In: Radiation-Induced Chromosome Damage in Man. T. Ishihara and M. Sasaki, eds. Alan R. I.iss, New York. pp. 215-316.

14. Bianchi MS, Bianchi NO, Idrramendy ML, García-Ileras J (1981): Chromosomal radiosensitivity of pig lymphocytes in relation to sampling time. Mutation Res 80:313-320.

15. Natarajan AT, Vyas RC; Wiegant J, Curado MP (1991): A cyto genetic follow-up study of the victims of a radiation accident in Goiania (Brazil). Mutation Res 247:103-111.

16. Heim S. Mitelman F (1995): Cancer Cytogenetics. 2nd Edition, Wiley-Liss, Inc.., Vew York.

17. Lichter P, Cremer 'T, Borden J, Manuelidis I., Ward DC: (1988): Delineation of individual human chromosomes in metaphase 
and interphase cells by in situ suppression hybridization using recombinant INA libraries. Hum Genet 80:224-234.

18. Pinkel D. Straume T, Gray JW (1986): Cytogenetic: analysis using quantitative, high sensitivity, fluorescence hybridization. Proc: Natl Acad Sci (USA) 83:2934-2938.

19. ISCN (1995): An International System for Human Cytogenetic Nomenclature. F. Mitelman. ed. Karger, S, Basel, pp. 1-114.
20. Savage JRK, Bigger TRI, (1978): Aberration distribution and chromosomally marked clones in X-irradiated skin. In: Mutagen-Induced Chronosome Damage in Man. H. I. Lvans and D. C.. Lloyd, eds. Edinburgh University Press. Edinburgh. p). $15 \overline{5}-169$.

21. Rabbitts TH (1994): Chromosomal tramslocations in human (ancer. Vature 372:143-149. 